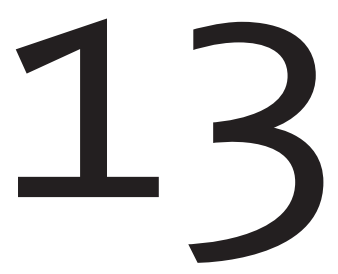

\title{
LA EVALUACIÓN EN ETAPAS ESCOLARES SIMILARES A LA E.S.O. EN ALGUNOS PAÍSES DE LA UNIÓN EUROPEA
}

\section{Assessment in Similar Levels as E.S.O. in some Countries of the European Union}

\author{
Ángel J. Rodríguez* \\ José M. Hernández" \\ José J. Castro*
}

\section{RESUMEN}

En este trabajo se estudian los procedimientos de calificación, organización de los equipos docentes, promoción, titulación, papel de la inspección educativa, recuperación de materias pendientes, uso de la evaluación inicial, de la evaluación y atención a la diversidad, etc. en las etapas educativas equivalentes a la Educación Secundaria Obligatoria (ESO) española, en Alemania, Inglaterra y Francia; a través del análisis de la práctica realizada en tres Colegios bilingües ubicados en la isla de Gran Canaria, mediante una entrevista semiestructurada realizada a sus directores y jefes de estudio, que siguen la normativa de sus respectivos países, así

* Universidad de Las Palmas de Gran Canaria. 
como del análisis de su legislación relativa a la evaluación. De esta comparación surgen algunas propuestas para optimizar la práctica educativa canaria y española en esta etapa educativa.

PALABRAS CLAVE: Evaluación, colegios bilingües, Educación Secundaria Obligatoria, España, Alemania, Francia, Inglaterra.

\section{ABSTRACT}

In this paper we study the qualification procedures, organization of teaching staff, promotion, certification, inspection role, pending recovery of materials, use of initial assessment, evaluation and attention to diversity, etc. in the stages of education equivalent to Spanish secondary education (ESO), Germany, England and France; through the analysis of the experience gained from three bilingual schools located in Gran Canaria which follow the rules of its own countries. From this comparison, some new proposals arise to optimize the education in the Canary Islands and Spain during this academic level.

KEY WORDS: Assessment, bilingual schools, Obligatory Secondary Education (ESO), Spain, Germany, France, England.

$$
* * * * *
$$

\section{INTRODUCCIÓN}

El marco educativo propuesto por las instituciones internacionales como la UNESCO y la Unión Europea propugna unas líneas comunes que permitan a los estados el logro de una educación capaz de formar ciudadanos libres y en igualdad de oportunidades. No obstante, la determinación de lo que es un ciudadano libre está indefectiblemente unida a concepciones culturales de todo tipo. La simple elección de un centro u otro, público o privado, laico o confesional, mono o plurilingüe puede suponer una diferencia sustancial en el tipo de persona que esa institución educativa esté contribuyendo a formar.

Si bien podría parecer que los modelos educativos de los países miembros de la Unión Europea son muy parecidos y que abordan los mismos problemas aportando soluciones similares, un minucioso análisis de sus propuestas curriculares permite reconocer múltiples diferencias, que bien aprovechadas pueden ayudar al enriquecimiento de todos, utilizando la 
educación comparada como medio para conocer y mejorar. En esta línea, se ha pretendido realizar un análisis comparativo de la educación secundaria de cuatro países de la Unión Europea: Francia, Inglaterra, Alemania y España, a partir de la propuestas curriculares concretas de centros de enseñanza de esta etapa, ubicados en Gran Canaria y que siguen los modelos educativos establecidos en sus respectivos países. En líneas generales debemos tener presente que nuestro sistema educativo suspende en calidad, eficacia y especialmente en competencias básicas (TORIBIO, 2008) y que precisa de iniciativas que lo mejoren.

¿Por qué elegir estos países y no otros?, pues porque los países de referencia han sido para España Francia con la LG-1970 e Inglaterra con la LOGSE-1990; de igual forma que Finlandia, por ejemplo, ha sido influenciada por los sistemas educativos de Alemania, la Unión Soviética y Suecia (GARCÍA, 2011).

En el análisis de las implicaciones de la globalización a la que están sometidos en la actualidad los sistemas educativos y socioeconómicos, cabe destacar en la educación (CARNOY, 1999: 145-162; PEDRÓ y ROLO, 1998: 266-271) el incremento del control sobre el currículo y la evaluación. Dentro del ámbito curricular, la evaluación nos permite conocer a qué aspectos se les concede especial importancia, precisamente en un período de crisis económica cuando las preocupaciones por la eficiencia se ponen de relieve con más facilidad y se trasladan al ámbito educativo donde consiguen una atención privilegiada (DURU-BELLAT, 2010), cómo deben ser adquiridos determinados contenidos o cómo debe ser demostrada dicha adquisición. En definitiva, permite ahondar en la perspectiva del sistema educativo de cada país a partir de su concepción de la educación. En esta línea se expresa el profesor Fernández Pérez (1998) cuando afirma que «todo lo que no se evalúa se devalúa», es decir, evaluamos aquello a lo que damos importancia y se la quitamos a lo que no evaluamos.

Los objetivos que persigue este trabajo son:

1. Conocer los procedimientos de calificación del rendimiento del alumnado.

2. Comparar el número de equipos docentes que se realizan en cada curso escolar. 
3. Analizar los procedimientos de promoción y titulación en su caso.

4. Acercarse a la función de la inspección educativa y de los consejos escolares respecto a la evaluación.

5. Conocer los procedimientos de recuperación de materias pendientes de cursos anteriores.

6. Acercarse a la práctica e interpretación de la evaluación inicial.

7. Comparar los procedimientos de evaluación con el alumnado de necesidades educativas especiales (NEAE).

8. Estudiar los procedimientos y regularidad en las formas e instrumentos de informar a las familias del rendimiento escolar de sus hijos.

9. Analizar los procedimientos de evaluación de la práctica docente y de las concreciones curriculares (antiguo PCC).

\section{MÉTODO}

En este estudio se compararon las propuestas de evaluación de tres centros de educación secundaria de países de la Unión Europea, concretamente Francia, Alemania y Reino Unido, en sus etapas educativas equivalentes a la E.S.O con el marco legislativo español. Los criterios de evaluación utilizados recogen la práctica de la evaluación en general: procedimientos de calificación, organización de los equipos docentes, promoción, titulación, uso de la evaluación inicial, de la evaluación y atención a la diversidad, etc. El método seguido para la realización del estudio comparativo de la evaluación en la educación secundaria ha pasado por las siguientes fases:

- Revisión pormenorizada de la legislación especifica sobre evaluación en etapas equivalentes a la Educación Secundaria Obligatoria de Francia, Alemania e Inglaterra, además de la española.

- Estudio sobre la forma de llevar a la práctica dicha normativa en centros educativos concretos. Esta fase estuvo centrada en una entrevista con los directores y/o jefes de estudio de tres centros cuyo 
funcionamiento sigue las directrices de cada uno de esos países. Dicha entrevista se sustentó en un cuestionario semiestructurado elaborado ad hoc a partir del estudio de la legislación de cada país.

- Análisis comparativo entre dichas propuestas y concreciones y el modelo de España. Debido a la organización autonómica del estado español, optamos por utilizar como referencia el marco legislativo vigente en la comunidad canaria puesto que, en líneas generales, puede usarse como ejemplo representativo, además de ser la comunidad donde llevamos a cabo nuestra labor docente.

- Establecimiento de propuestas de mejora. La parte final del trabajo pretende sugerir algunas consideraciones a tener en cuenta para mejorar la evaluación en España a partir del cotejo con los otros modelos.

En cuanto a la muestra de centros considerados, estuvo formada por el Colegio Francés de Telde «René Verneau»; el Colegio Oficial Alemán de Las Palmas, que sigue las directrices del Länd de Hamburgo, y el Oakley College, con un sistema basado en el de Inglaterra y Gales, todos enclavados en la isla de Gran Canaria.

El método de investigación es indudablemente descriptivo, dentro del paradigma orientado a la decisión y al cambio. Como afirma Del Río Sadornil (2003:112), donde «tienen cabida los estudios tipo encuesta, basados en la recogida de información rica y variada sobre grupos de sujetos que sirvan de base para la toma de decisiones».

\section{ANÁLISIS COMPARATIVO}

La ubicación y estructura de la enseñanza secundaria obligatoria en el sistema educativo de cada país responde a una larga tradición: mientras en Francia, como en España, los alumnos están escolarizados doce cursos antes de la universidad, en Alemania e Inglaterra lo hacen durante trece años; si bien están en vías de reducir la escolaridad a doce.

La duración de la educación secundaria obligatoria es variable: cuatro años en España Francia, cinco en Inglaterra y seis en el nivel secundario I de Alemania (se trata de un gymnasium, tipo de centro que permite asistir a la 
universidad). El sistema inglés es casi idéntico al español: seis años de primaria y dos de bachillerato.

\subsection{Evaluación inicial}

En Francia e Inglaterra cuentan con Exámenes de Estado al comienzo de la etapa, siendo los únicos países que disponen de estas pruebas al entrar en la secundaria obligatoria. Además de esto, en el ecuador de la etapa en el sistema británico, hay otra vez pruebas de estado al comenzar el curso. En España existen las Pruebas de Diagnóstico, que con la LOCE se aplicaban en $4^{\circ}$ y con la LOE en $2^{\circ}$ de la ESO.

En el sistema británico no suelen utilizar pruebas de evaluación inicial, sino que los y las profesoras confían y parten del resultado de las pruebas finales del curso precedente.

En cualquier caso en todos los países cada profesor en concreto hace uso de sus propias pruebas de evaluación inicial; no como en España, donde cada Departamento Didáctico suele disponer de las propias y ser comunes para todos los profesores que impartan clase en un mismo nivel.

En definitiva, no es obligatoria la evaluación inicial en ningún país, aunque es recomendada.

\subsection{Procedimientos de calificación en la etapa equivalente a la E.S.O.}

Las escalas que se utilizan para calificar son radicalmente diferentes: numéricas del 0 al 20 en Francia (10 ó más es aprobado); Alemania usa una escala numérica del 1 al 6 (1 -sehr gut- muy bien, 2 - gut-bien, 3befriedigend-razonable, 4 -ausreichend-suficiente-, 5 -mangelhaftdeficiente, 6-ungenügend-insuficiente); Inglaterra aplica la siguiente escala de letras: A*-A-B-C-D-E (la mejor es A*); En España se usa la escala numérica del 1 al 10 .

Como se ve, responden a las tradiciones de cada país y no han cambiado, excepto en España, en los últimos años. 
En el boletín informativo sobre las calificaciones, ningún país suele utilizar otros indicadores tales como capacidades o competencias básicas (CCBB), excepto en Canarias donde es obligatoria hacerlo desde el curso 2011.12, pero sí introducen observaciones a las mismas; para evaluarlas entre todos los miembros del equipo docente cada centro ha elaborado procedimientos diferentes: en ocasiones cada departamento didáctico sólo puntúa 5 de las 8 , en otras ocasiones han elaborado una sofisticada hoja de cálculo que da una ponderación, según la materia, a cada competencia (RODRÍGUEZ, 2011). No obstante, siendo los criterios de evaluación el referente fundamental para la evaluación del rendimiento del alumnado, la ligazón entre estos y las CCBB sigue siendo confusa: la Consejería de Educación del Gobierno de Canarias está elaborando este curso 2011.12 rúbricas que ayudan a enlazar estos dos elementos curriculares.

Un aspecto a tener muy en cuenta es la «nota oral» alemana que valora el trabajo en clase, interés por la materia, respeto, realización de deberes, etc. en un 50\% de la calificación final; algo parecido se hace en Francia e Inglaterra (excepto en el Y11, equivalente a $4^{\circ}$ de la ESO, pues se centran en cuestiones conceptuales y procedimentales para preparar el examen de Estado que les permitirá en su caso cursar bachillerato), pero no lo tienen estipulado en un porcentaje concreto.

Respecto a la nota final de curso, en Inglaterra depende de cada centro el que sea evaluación continua, un solo examen u otra opción, en Alemania se valoran los dos semestres, si bien con más fuerza y peso el segundo; quizás la aportación más interesante sea la francesa, donde valoran la tendencia de mejora o empeoramiento.

En ningún país utilizan una fórmula matemática del tipo media ponderada o media aritmética, si bien en general el peso de la última evaluación es sumamente importante.

La autoevaluación y la coevaluación se utilizan más en el sistema francés y especialmente en el alemán que en el británico. En España no se utiliza generalmente de acuerdo con los datos de este estudio. Lo mismo ocurre con la evaluación individualizada, se utiliza en una mayor medida en Francia y Alemania que en Inglaterra, según lo manifestado por sus directores y jefes de estudio. 
En cuanto a las evaluaciones que tienen por curso, Francia e Inglaterra coinciden con Canarias: tres por curso, Alemania tiene sólo dos. Cabe resaltar la particularidad de que en Inglaterra depende de cada centro, quienes de hecho podrían optar por tener una sola al año.

\subsection{Los equipos educativos o sesiones de evaluación de los equipos docentes}

Respecto al número de equipos educativos el sistema francés es similar al español: prescribe que sean cinco como mínimo, en la comunidad canaria son al menos cinco como mínimo según la legislación LOE. En Alemania hay dos o más y en Inglaterra no está regulado, sino que depende de cada centro.

La coordinación de estos equipos educativos la hace en Francia y Alemania el tutor, lo mismo que en España. En Inglaterra es el jefe de Estudios. La participación de los alumnos en los equipos educativos sólo se realiza en Francia, al igual que en España; no participan los alumnos ni en Alemania ni en Inglaterra.

\subsection{Criterios de promoción}

La permanencia más de un año en un mismo curso es una cuestión sumamente controvertida. Si bien se puede repetir curso en los cuatro países objeto de estudio, hacerlo en el sistema británico es algo extraordinario.

Los criterios de promoción alemanes eran similares a los españoles en la LOCE, que son fijos y por asignaturas: con más de dos asignaturas se repite, aunque hay variaciones según el Länd; si bien en España con la LOE se pasa con una o dos materias suspensas y con tres vota el equipo docente, según haya alcanzado o no las CCBB, entre otras cuestiones a considerar. En Inglaterra depende de cada centro y en Francia hacen una media de las calificaciones de las asignaturas que debe ser como mínimo de 10 (su aprobado); en todo caso valoran la tendencia del alumno, si está mejorando o empeorando, edad del sujeto, etc. Los equipos docentes se adaptan a cada alumno en concreto. 
En España y Alemania un equipo educativo no puede incumplir los criterios de promoción, en Francia no hay criterios y en Inglaterra pueden hacerlo si tienen el beneplácito de la Jefatura de Estudios. Si bien en nuestro país en numerosas ocasiones el profesorado cambia sus calificaciones, para adaptarse a la norma y promocionar al alumno en su caso.

La opinión de la familia es tenida en cuenta en Francia, pero la decisión final es del director; en Inglaterra es muy tenida en cuenta; en Alemania y España la opinión de los padres es poco relevante para la promoción en la educación secundaria elemental.

En España se puede repetir una vez cada nivel con la LOE, lo mismo que en Alemania; en este país si se repite un nivel, no se podrá repetir el nivel siguiente, mientras que en España sí. En Francia e Inglaterra no hay límite al respecto.

Los alumnos que están repitiendo suelen llevar refuerzos o apoyos en Francia, España e Inglaterra, aunque depende de las posibilidades del centro; no reciben ayuda extra en Alemania.

\subsection{Procedimiento de titulación}

Cabe mencionar que sólo se obtiene título en Francia y en España. Tanto en Francia como en Inglaterra, al terminar la Etapa los alumnos deben realizar un Examen de Estado, supervisado por un Inspector; dicho examen es corregido a nivel central, aspecto que permite al Estado conocer el nivel de eficacia de su Sistema Educativo e igualar los criterios de titulación para todos los alumnos del país.

En Francia para obtener el «Diplôme Nationalle de Brevet» deben tener al menos un 10 de media entre todas las asignaturas (sistema similar al de la promoción). Por otra parte, parece muy interesante la propuesta gala de que además del número de asignaturas suspensas se tenga en cuenta la media obtenida.

En Alemania no pueden tener más de dos asignaturas suspensas para pasar al bachillerato, mientras que en Inglaterra deben aprobar un mínimo de 4 de las 10 asignaturas en su examen de Estado; con mucho el país más benigno para poder cursar bachillerato. 
Todos valoran las asignaturas, ninguno se basa sólo en una apreciación de capacidades o competencias básicas, si bien las tienen en cuenta dentro de la valoración de cada una de ellas. Sólo en España, con la LOCE, se valoraba de forma especial las Matemáticas y la Lengua Castellana, peculiaridad anulada con la LOE.

En cuanto a los alumnos con necesidades educativas especiales (n.e.e.) tienen los mismos procedimientos para titular o pasar a bachillerato que el resto de los alumnos en Alemania, Inglaterra y España; sólo en Francia articulan procedimientos específicos de acceso. En todos los países son pocos los alumnos con n.e.e. que obtienen el título o promocionan a bachillerato, especialmente si son de origen cognitivo (caso aparte si son nee de origen sensorial).

\subsection{Papel de la Inspección y del consejo escolar}

El colegio Francés «René Verneau» y el Alemán tienen Inspección de su país respectivo, el Oakley College tiene inspección española y británica.

En general la presencia de la Inspección en Francia, Alemania e Inglaterra es mayor que en España. En estos países los inspectores pasan clase por clase cada año, supervisan las pruebas finales de cada curso, asesoran y hacen seguimiento. Todo ello en nuestro país se hace a un nivel mucho menor si exceptuamos los centros sometidos al plan de evaluación del Instituto de Evaluación, con sus sucursales en cada comunidad autónoma, como es el caso del Instituto Canario de Evaluación y Calidad (ICEC), en los cuales el control y el asesoramiento es exhaustivo. En los tres países existe un organismo similar al Consejo Escolar, quien analiza y hace sugerencias sobre los resultados de la evaluación. Si bien en España lo hacen de forma superficial y con muy poca trascendencia real al claustro de profesores.

\subsection{Las reclamaciones a las calificaciones y a la promoción/titulación}

En todos los países se puede reclamar, tanto a las calificaciones en un área concreta como a la decisión de promocionar o titular en su caso. Las reclamaciones son muy poco numerosas y suelen referirse a la coherencia 
entre las calificaciones obtenidas a lo largo del curso y la nota final, así como a la corrección de determinados exámenes.

Cuando la reclamación se refiere a la calificación de una asignatura concreta, en Francia y Alemania decide el propio profesor si cambia la nota o no, en Inglaterra es el Director y en España el Departamento Didáctico implicado.

Si la reclamación se refiere a la promoción o titulación en su caso, en Alemania decide el Director, en Francia una comisión de profesores y el Director, en Inglaterra el Director y el Consejo Escolar y en España el equipo docente en primera instancia y el Director o el Inspector en segunda $\mathrm{y}$ tercera instancias.

\subsection{El consejo orientador}

Todos los países utilizan algún tipo de consejo orientador para el alumnado al finalizar la Etapa, pero la diferencia está en que Alemania lo entrega tras el primer año de secundaria, para ver en qué tipo de centro de secundaria continúa sus estudios, mientras que en España, Francia e Inglaterra se entrega al final del último curso. En Francia y Alemania en junio, en España en marzo/abril y en Inglaterra en agosto, cuando tienen los resultados del Examen de Estado.

El contenido del consejo orientador lo deciden en Inglaterra y Alemania el equipo docente, al igual que en nuestro país. En Francia lo hace el Consejo de Orientación, compuesto por el tutor, los padres y el director. En España lo decide el tutor con la LOCE y el Equipo Docente con la LOE.

En Inglaterra y España el consejo orientador no es de obligado cumplimiento por parte del alumnado, en Alemania y Francia sí.

\subsection{Recuperación de áreas y materias pendientes}

Ningún país, excepto España, utiliza exámenes finales, pruebas extraordinarias de recuperación. Todos recuperan las pendientes mediante la observación de la evolución del rendimiento del alumno en la asignatura 
homónima o de continuidad. Si no cursan homónima las pendientes de otros cursos no se tienen en cuenta a la hora de titular o promocionar en los tres países estudiados.

Esto va en contra de la tradición española; nuestra polémica de exámenes de recuperación en junio o septiembre no existe en estos países europeos.

\subsection{Evaluación y atención a la diversidad}

En todos los países hay niños con n.e.e. integrados en centros ordinarios, si bien en Francia no todos los centros son de integración, tal como ocurre en determinadas Comunidades Autónomas como por ejemplo Castilla y León en España.

La calificación de estos alumnos la hacen de forma diferente: en Francia a lo largo del curso es el profesor de apoyo a las n.e.e. quien decide la calificación, pero la nota final la hace el equipo educativo. En Alemania siempre son calificados por el profesor ordinario, como en teoría ocurre en España (aunque la Resolución de atención al alumnado con NEAE BOC del 24 de febrero de 2011 en Canarias sugiere que esta calificación sea consensuada entre el profesor de apoyo a las NEAE y el de área). En Inglaterra son ambos, el de apoyo y el ordinario de cada materia quienes califican a los alumnos con n.e.e.

El alumnado con n.e.e. tiene los mismos criterios de promoción y titulación que el resto de sus compañeros, aunque en Francia pueden tener pruebas especializadas, tal como se comentó anteriormente y en España no pueden promocionar de $2^{\circ}$ a $3^{\circ}$ de la ESO con la LOE, si no han superado la Primaria.

\subsection{Información a la familia}

Es obligatorio informar a las familias al principio de curso sobre los criterios de evaluación, promoción, titulación etc., si bien en Francia sólo es obligatorio hacerlo a los alumnos de integración y en Inglaterra sólo de forma oral. En España, concretamente en Canarias, es preciso informar a la familia de forma oral y tenerlos expuestos a su disposición, aspecto que no 
siempre se cumple y que no se contempla en la normativa que desarrolla la LOE, a pesar de que sí lo era en la normativa LOCE (2002) y LOGSE (1990).

Por lo que respecta a boletines informativos a las familias hay tres en todos los países, excepto en Alemania, donde son dos.

Las reuniones colectivas con los padres de alumnos son dos como mínimo en España y en Francia, una en Alemania y ninguna en el sistema inglés. Las reuniones individuales son nueve como mínimo en Canarias; en Francia e Inglaterra no hay mínimo, tienen que solicitar los padres la visita; en Alemania tienen un día a la semana para este tipo de visitas.

En todos los países los padres tienen que solicitar con antelación al tutor las entrevistas, para que éste pueda recabar información de todo el equipo docente.

\subsection{Evaluación de la práctica docente y del proyecto curricular}

Francia tiene un currículo para todos los centros del país y en todos es idéntico. En Alemania el currículo es el mismo para todos los centros de cada Länd; en ambos casos no hay proyecto curricular de centro (P.C.C., Concreción curricular en terminología LOE) propio. Sólo en Inglaterra tienen un sistema similar al español: un currículo por Estado (Comunidad Autónoma) adaptado por el profesorado a cada instituto en concreto.

La evaluación de la práctica docente la efectúan los propios directores en Inglaterra y Francia; en Alemania, como en España, son los propios profesores, con la particularidad de que en Alemania se aplica al contar con «días pedagógicos» destinados a ello y en España no se aplica con mucha regularidad.

Instrumentos y procedimientos para evaluar el PCC sólo pueden tener los ingleses, ya que son los únicos que cuentan con este documento y efectivamente disponen de ellos.

Todos los países evalúan, al menos de forma teórica, las relaciones entre los miembros de la comunidad escolar. 


\section{CONCLUSIONES}

Este apartado del trabajo se ha centrado en aquellos aspectos más significativos $\mathrm{y}$, sobre todo, en los puntos que han emergido como potenciales contribuciones a la mejora del sistema educativo español.

Respecto a las escalas utilizadas en el boletín informativo de las calificaciones obtenidas por los estudiantes, todo parece aconsejar que se utilice en nuestro país la escala tradicional del 0 al 10, para no confundir a la comunidad educativa. La escala promulgada en la Orden de 7 de noviembre de 2007, por la que se regula la evaluación del alumnado de enseñanza básica en Canarias (BOC nº 235 de 23 de noviembre de 2007) y que desarrolla la LOE, del 1 al 10, puede ser igualmente válida. Utilizar cualquier otra, sean letras o de cualquier otro tipo, no parece aportar las mejoras suficientes que compensen la desorientación que produce su incorporación entre la comunidad educativa, tal como se ha apreciado en los años precedentes con la LOGSE. Nos parece muy loable la normativa española que prescribe la calificación de las CCBB, aunque quizás deberían haber aportado antes documentos que las ligaran a los criterios de evaluación y a los instrumentos de calificación utilizados tradicionalmente por el profesorado en esta etapa: actitud, cuaderno, trabajos, trabajo en equipo, controles, preguntas de clase, etc.

Para los padres es muy ilustrativo que haya observaciones sobre las calificaciones obtenidas por sus hijos de forma escrita; pero esto no siempre se realiza. Su presencia en los tres países considerados sugiere que quizás, la normativa española debería recoger la obligatoriedad de, ante una nota desfavorable, informar a los padres sobre las causas de la misma y, si cabe, de las actividades recomendadas para superarla. Siempre es mejor entregar unas observaciones con recomendaciones que sólo utilizar indicadores de capacidades o competencias básicas que en numerosas ocasiones han resultado enigmáticas e ininteligibles a las familias.

Un aspecto a tener muy en cuenta es la «nota oral» alemana que valora el trabajo en clase, interés por la materia, respeto, deberes etc., en un 50\% de la calificación final; algo parecido se hace en Francia e Inglaterra aunque no esté estipulado en un porcentaje concreto. En Canarias se podrían publicar unas recomendaciones para valorar los aspectos integrados en la «nota oral» 
alemana, pues en la actualidad se hace, pero no en todos los centros ni en todos los departamentos didácticos por igual.

Respecto a la aplicación de la evaluación continua en el momento de establecer la nota final de curso en junio, resulta muy interesante el procedimiento de Francia, que valora la tendencia y la evolución del alumno en cada área, sin necesidad de recurrir a medias ponderadas ni a utilizar exclusivamente la nota del tercer trimestre, medidas ambas cargadas de inconvenientes.

Se echa en falta una mención a la autoevaluación y a la coevaluación en las normativas de las diversas comunidades autónomas españolas, como medio de desarrollo personal del alumno así como favorecedor de su integración en su proceso educativo y de la cohesión grupal. Otro tanto se podría afirmar sobre la evaluación individualizada y criterial.

En lo que atañe al número de sesiones de evaluación del alumnado, equipos educativos, creemos que el número de tres en cada curso escolar es inadecuado por escaso. Cinco sesiones de evaluación del equipo docente como mínimo, tal como indica la normativa canaria que desarrolla la LOE pueden ser suficientes: uno a finales de septiembre para conocer el grupo e intercambiar información sobre las características psicopedagógicas y primeras impresiones, otro en diciembre para las calificaciones del primer trimestre, otro al final del segundo trimestre para dar consejo orientador, derivar al alumnado a ciclos formativos o PCPI en su caso y calificaciones del segundo trimestre, otro a final de junio y el quinto en septiembre. No obstante hay que señalar que se suelen reunir más veces para tratar cuestiones extraordinarias: casos puntuales de convivencia, medidas de atención a la diversidad, etc.

La evaluación de la práctica docente en España prácticamente no se aplica nunca. A lo sumo se desarrolla este apartado en el PCC (denominado «concreción curricular» en la LOE), pero en realidad nunca se llega a poner en práctica y menos aún se hace de forma regular. Por todo ello los «días pedagógicos» alemanes, jornadas dedicadas a ello en exclusividad, serían una iniciativa interesante; aunque esto supondría romper con la tradición del profesorado español de tener vacaciones todo el mes de julio. 
Los criterios para promocionar o no en las etapas equivalentes a la ESO son muy variados; en todo caso la simbiosis del sistema alemán por asignaturas con el francés, según la tendencia de cada alumno, de alguna forma queda plasmado en la LOE al repetir si suspenden cuatro materias, pasar si sólo suspenden una o dos y al estudiarse si las suspensas son tres (debiendo votar favorablemente al menos la mitad del equipo docente). La práctica alemana consistente en que si se repite un curso no se puede repetir el siguiente nos parece muy interesante, para evitar aglomeración de alumnos, mayores y frustrados en $1^{\circ}$ y $2^{\circ}$ de la ESO con los problemas de conducta que esto favorece. De aplicarse tendríamos la ventaja de que un alumno tras repetir $1^{\circ}$ de la ESO no podría volver a repetir hasta $3^{\circ}$ de la ESO y evitaríamos el colapso actual en los dos primeros niveles de la etapa, además de ahorro económico que supondría para la Administración Educativa disminuir el número de repeticiones. A pesar de que la inmensa mayoría del profesorado está de acuerdo con la posibilidad de repetir en cualquier nivel de la ESO (RODRÍGUEZ, 2009).

Todos los países estudiados otorgan el título en base a las asignaturas superadas, sin dejar de tener en cuenta otros aspectos, como los objetivos de la etapa y las competencias básicas. Por ello se propone un sistema para la titulación similar al legislado para la promoción, que se titule con una o dos suspensas, no se pueda hacer con cuatro y que con tres se valoren capacidades, competencias básicas, la media obtenida, etc. tal como ha legislado la Orden de 7 de noviembre de 2007, por la que se regula la evaluación del alumnado de enseñanza básica en Canarias (BOC nº235 de 23 de noviembre de 2007).

Respecto a la titulación otorgada a los alumnos con n.e.e. en la actualidad en España es prácticamente imposible. Dentro de las soluciones que se plantean estarían: a) Tener en cuenta la nota media como en Francia y, b) hacer pruebas específicas para ellos, totalmente diferentes.

Una buena solución podría ser la unión de ambas propuestas: que titulen, todos los alumnos, siempre que tengan un cinco de media y a juicio del equipo docente hayan adquirido las competencias básicas y los objetivos mínimos de la etapa, aunque haya más de dos suspensas. Lo mismo podría hacerse con la promoción, pues en la actualidad es difícil que un alumno con n.e.e. no repita cada uno de los niveles y pase de cursar $2^{\circ}$ de la ESO, por lo 
que se desmoraliza al verse mucho mayor que sus compañeros y no tener ninguna oportunidad de titular.

En cuanto al papel de la Inspección, es posible que sigamos pagando algún tipo de tributo por la práctica de la misma con la LGE de 1970, donde su presencia era cercana y se vivía de forma casi amenazante por el profesorado; quizás para compensar esta práctica histórica, en la actualidad contamos con una Inspección Educativa mucho más alejada de su trabajo de supervisión, control y asesoramiento que en el resto de Europa. En nuestra opinión ha llegado la hora de liberarnos de nuestras cargas históricas y prejuicios y atrevernos a apostar por una Inspección más cercana a cada centro educativo y con un enfoque más orientador.

$\mathrm{El}$ consejo orientador que se entrega en $4^{\circ}$ de la ESO, parece razonable que se retrase de marzo a junio, para evitar dar un consejo sin tener en cuenta el último trimestre, que en algunos casos es decisivo y testigo de un cambio importante en el rendimiento escolar del alumnado. No somos partidarios de que el consejo orientador sea de obligado cumplimiento, como ocurre en Francia y Alemania. La práctica alemana de dar el consejo orientador tras el $1^{\circ}$ de la ESO (algo similar contemplaba la LOCE-2002), merecería ser estudiada y quizá implementada con centros piloto y estudios a medio y largo plazo para analizar sus resultados.

Respecto a los exámenes de recuperación de pendientes, las pruebas extraordinarias reinstauradas por la LOCE, no se utilizan en el resto de los países estudiados. En el caso de que debieran existir estos exámenes, tendrían que celebrarse en septiembre y no sólo en $4^{\circ}$ de la ESO como propugna la LOE, sino en los cuatro cursos de la etapa. Además deberían entregarse unas actividades individualizadas que tuvieran un valor aproximado del $50 \%$ de la calificación; de forma similar a como prescribía, aunque no se cumplió, la LGE (1970).

En cuanto a la evaluación del alumnado con n.e.e., creemos que debería figurar explícitamente en la normativa que sea el profesor ordinario el responsable de poner la calificación, pero contando con la opinión del profesor de apoyo a las n.e.e., ya que en la actualidad es casi siempre el profesor de apoyo el único que califica a este alumnado. Esta práctica no ayuda a responsabilizarse de este de alumnado al profesorado ordinario. 
A continuación se presenta una tabla que recoge los aspectos más destacados del estudio comparado de la evaluación en los centros considerados.

Tabla 1. Comparativa de los principales apartados de la evaluación

\begin{tabular}{|c|c|c|c|c|c|c|c|c|c|c|c|c|c|c|c|c|}
\hline Países & \multicolumn{16}{|c|}{ CRITERIOS } \\
\hline & 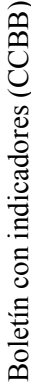 & 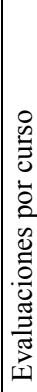 & 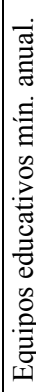 & 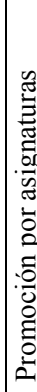 & 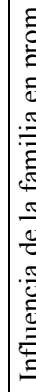 & . & 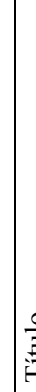 & & & 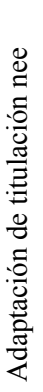 & 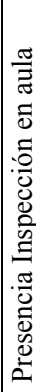 & 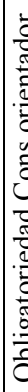 & & 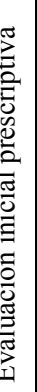 & 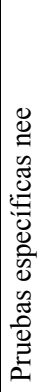 & 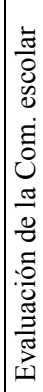 \\
\hline Alemania & & 2 & 2 & & & & & & & & & & & & & \\
\hline Inglaterra & & 3 & & & r & & & & & & & & & & & 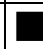 \\
\hline Francia & & 3 & 5 & & - & & & & & & & & & & & \\
\hline España & & 3 & 5 & & & & & & & & & & & & & \\
\hline
\end{tabular}

* NOTA: cuando aparece un recuadro negro indica que ese elemento está presente en la evaluación del país en cuestión. El número indica la cantidad de veces que se produce durante el año.

\section{REFERENCIAS BIBLIOGRÁFICAS}

CARNOY, M. (1999): Globalización y reestructuración de la educación, Revista de Educación, 318, pp. 145-162.

DURU-BELLAT, M. (2010): Las desigualdades educativas en Europa: una cuestión de actualidad, Revista Española de Educación Comparada, 16, pp. 105-130.

GARCÍA GARRIDO, J. L. (1993): Sistemas educativos de hoy (3ª edición) (Madrid, Dykinson).

GARCÍA RUIZ, $\mathrm{M}^{\mathrm{a}}$ J. (2011): Contrastes de las culturas escolares finlandesa y española, Revista Española de Educación Comparada, 18, pp. 157-202.

FERNÁNDEZ PÉREZ, M. (1998): Evaluación y cambio educativo (5 $5^{a}$ edición) (Madrid, Ediciones Morata). 
DEL RÍO SARDONIL, D. (2003): Modelos de Investigación en Educación (Vol I) (Madrid, UNED).

PEDRÓ, F. and ROLO, J. M. (1998): Los sistemas educativos iberoamericanos en el contexto de la globalización. Interrogantes y oportunidades, Revista Iberoamericana de Educación, 17, pp. 257-289.

RODRIGUEZ. FDEZ. A. J. (2009): Metaevaluación de la ESO en Canarias: análisis y propuestas (Las Palmas de Gran Canaria, Tesis doctoral).

TORIBIO BRIÑAS, L. (2008): Un análisis del sistema educativo español desde la perspectiva comparativa europea, Tendencias Pedagógicas, 13, pp. 39-58.

RODRÍGUEZ FDEZ. A.J. y HERNÁNDEZ PÉREZ, J.M (2011): Evaluación de las CCBB en la Enseñanza Obligatoria (Bragança:Conferencia Ibérica ieTIC. IP Bragança y Universidad de Salamanca).

\section{REFERENCIAS LEGALES (POR PAÍSES)}

\section{Alemania}

Bekanntmachung vom 9. Dezember 2003, GABI. 2/2004 S.34

Zuletzt gaädert durch Bekanntmachung vom 10. November 2004, ABI. 12/2004

\section{España}

LEY 14/1970, de 4 de agosto, General De Educación y Financiamiento De La Reforma Educativa (BOE n ${ }^{\circ} 187$ de 6 de agosto de 1970).

Decreto 2618/1970, de 22 de agosto, sobre sustitución de las actuales pruebas del grado de bachiller elemental y establecimiento de la evaluación continua del rendimiento educativo de los alumnos (BOE $n^{\circ} 225$ de 19 de septiembre de 1970).

Orden de 16 de noviembre 1970 sobre evaluación continua del rendimiento educativo de los alumnos ( $\mathrm{BOE} \mathrm{n}^{\circ} 282$ de 25 de noviembre de 1970).

Resolución de 17 de noviembre de la Dirección General de Enseñanza Media y Profesional por la que se dan instrucciones para la aplicación de la evaluación continua del rendimiento educativo en los centros de Enseñanza Media. (BOE $n^{\circ}$ 282 de 25 de noviembre de 1970)

Resolución de la Dirección General de Enseñanza Primaria por la que se dan instrucciones sobre evaluación continua en la Educación General Básica. (BOE $\mathrm{n}^{\mathrm{o}} 289$ de 3 de diciembre de 1970). 
Orden de 12 de mayo de 1971 por la que se regula la homologación del acta final en la evaluación continua (BOE $n^{\circ} 127$ de 28 de mayo de 1971).

Orden de 12 de mayo de 1971 por la que se regulan las pruebas de conjunto que sustituyen a las de grado del Bachillerato Elemental para los alumnos no sometidos al régimen de evaluación continua (BOE $n^{\circ} 127$ de 28 de mayo de 1971).

Orden de 14 de febrero de 1972bpor la que se dictan normas para la evaluación continua del rendimiento educativo de los alumnos de Formación Profesional. (BOE n ${ }^{\circ} 59$ de 9 de marzo de 1972).

Resolución de la Dirección General de Ordenación Educativa por la que se dan instrucciones para la realización de pruebas flexibles de promoción en la segunda etapa de educación General Básica. (BOE nº 113 de 11 de mayo de 1973).

Orden de 2 de julio de 1974 por la que se complementa la de 16 de noviembre de 1970 sobre evaluación continua del rendimiento educativo de los alumnos. (BOE $\mathrm{n}^{\circ} 158$ de 3 de julio de 1974)

LOGSE Ley Orgánica 1/1990, de 3 de octubre de 1990 de ordenación General del Sistema Educativo (BOE n⿳0 238 de 4 de octubre de 1990).

Orden de 13 de mayo de 1993, sobre Evaluación en Educación Secundaria Obligatoria en Canarias (BOC nº71 de 2 de junio de 1993).

Orden de 3 de abril de 1995 sobre Evaluación en Bachillerato (BOC n ${ }^{\circ} 143$ del 21 de abril de 1995). (matizada en la Orden de 1 de Junio de 1999 en el BOC del 7 de junio de 1999).

LOPEG Ley Orgánica 9/1995, de 20 de noviembre de la participación, la Evaluación y el Gobierno de los Centros Docentes (BOE n 278 de 21 de noviembre de 1995).

Resolución de 29 de abril de 1996 de la Dirección General de Centros Escolares sobre la Organización de los Departamentos de Orientación en los Institutos de Educación Secundaria (BOE no 132 de 31 de mayo de 1996).

Orden de 7 de abril de 1997 por la que se regula el procedimiento de realización de las ACI (BOC nº 45 de 25 de abril de 1997).

Orden de 9 de abril de 1997 sobre la escolarización y recursos para alumnos con n.e.e. (BOC n ${ }^{\circ} 45$ de 25 de abril de 1997).

Orden de 13 de agosto de 1998, por la que se aprueban las Instrucciones de organización y funcionamiento de los Institutos de Educación Secundaria 
dependientes de la Comunidad Autónoma de Canarias (BOC n 109 de 26 de agosto de 1998).

Orden de 8 de marzo de 1999 que regula la escolarización (B.O.C. $\mathrm{n}^{\circ} 44$ de 17 de marzo de 1999).

Orden de 13 de mayo de 1999, sobre evaluación en la Educación Secundaria Obligatoria (BOC $\mathrm{n}^{\mathrm{o}} 72$ de 7 de junio de 1999).

Ley Orgánica 10/2002, de 23 de diciembre, de Calidad de la Educación. (BOE n ${ }^{\circ}$ 307 de 24 de diciembre de 2002).

Resolución de 20 de maig de 2003, que dóna instruccions per a l'organització i el funcionament dels centres d'education secundària de catalunya per el curs 20032004 (Diario Oficial de Cataluña no 961 de 21 de junio de 2003).

Real decreto 831/2003, de 27 de junio, por el que se establece la ordenación general y las enseñanzas comunes de la E.S.O. (BOE n ${ }^{\circ} 158$ de 3 de julio de 2003).

Orden EDC/1923/2003 de 8 de julio, por la que se establecen los elementos básicos de los documentos de evaluación, de las enseñanzas escolares de régimen general reguladas por la Ley Orgánica 10/2002, de 23 de diciembre, de Calidad de la Educación, así como los requisitos formales derivados del proceso. de evaluación que son precisos para garantizar la movilidad de los alumnos. (BOE $\mathrm{n}^{\circ} 165$ de 11 de julio de 2003).

Orden ECD 2286/2003, de 31 de julio (B.O.E. nº 192 de 12 de agosto).

Orden do 25 de agosto de 2003 (Diario Oficial de Galicia n ${ }^{\circ} 178$ de 15 de setembre $\left.\mathrm{n}^{\circ} 178\right)$.

Orden 4997/2003, de 3 de septiembre, del Consejero de Educación para la aplicación en el curso académico 2003-2004, de la evaluación, promoción y titulación en la E.S.O. reguladas en la Ley orgánica 10/2002, de 23 de diciembre, de Calidad de la Educación. (Boletín Oficial de la Comunidad de Madrid BOCM nº 213 de 8 de septiembre de 2003).

Orden de 13 de septiembre de 2003, por la que se establecen nuevos criterios de evaluación, promoció y titulación en la E.S.O. (BORM n 246 de 23 de octubre de 2003), (C.A. de Murcia).

Orden EDU/1186/2003, de 15 de septiembre, por la que se modifica la Orden de 29 de abril de 2002, de la Consejería de Educación y Cultura, por la que se regula la impartición dela E.S.O. en Castilla y León. (BOC y L nº 180 de 17 de septiembre de 2003). 
Orden de 1 de octubre de 2003, por la que se establecen criterios para la aplicación de la evaluación y promoción, y requisitos para la titulación del alumnado que curse Educación Secundaria Obligatoria. (BOC n ${ }^{\circ} 198$ de 10 de octubre de 2003).

Orden de 3 de Octubre de 2003 (DOGV no 4.624 de 6 noviembre, C.A. de Valencia).

Instrucciones del director general de ordenación, Renovación y Centros de 17 de octubre de 2003 (No publicadas en el DOE, Extremadura).

Orden Foral 452/2003, de 17 de octubre (BON n ${ }^{\circ} 145$ de 14 de noviembre de 2003), (Navarra).

Orden de 21 de octubre de 2003 (BOIB n ${ }^{\circ} 153$ de 4 noviembre de 2003), (C. A. de Baleares).

Resolución de 31 de octubre de 2003 por la que se dictan instrucciones sobre evaluación, promoción y titulación en la E.S.O. (B.O.P.A.N. no 264 de 14 de noviembre de 2003), (C. A. de Asturias).

Instrucciones del 4 de noviembre de 2003 del Viceconsejero de Educación sobre las condiciones de evaluación, promoción y titilación en la E.S.O. para el curso 2003-2004 (País Vasco).

Resolución de 10 de noviembre de 2003, por la que se dictan instrucciones para la aplicación en el curso académico 2003-2004 de la Evaluación, Promoción y Titulación en la E.S.O. reguladas en la Ley Orgánica 10/2002, de Calidad de la Educación (BOC nº 224 de 20 de diciembre de 2003), (C.A. de Cantabria).

Resolución de 17 de noviembre de 2003, de la Dirección General de Ordenación Educativa y Formación Profesional en la que se dan instrucciones sobre la evaluación, promoción y de los requisitos para la obtención del título de Graduado en Educación Secundaria Obligatoria para el curso 2003-2004 (DOCM n 167 de 28 de noviembre de 2003), (C.A. de Castilla La Mancha).

Instrucciones de 21 de noviembre de 2003, de la Dirección General de Evaluación Educativa y Formación del profesorado, por las que se dictan normas para la aplicación de lo establecido en los artículos 28, 29 y 31.2 de la Ley orgánica 10/2002, de 23 de diciembre, de Calidad de la Educación. (C.A. de Andalucía)

Orden 52/2003 de 27 de noviembre (Boletín Oficial de la Rioja n 147 de 29 de noviembre de 2003).

Resolución de 28 de noviembre de 2003, de la Dirección general de Política Educativa por la que se dictan instrucciones sobre evaluación, promoción y titulación en la E.S.O. para el curso 2003/2004 (BOA no 34 de 5 de diciembre de 2003). (C.A. de Aragón). 
Resolución de 20 de octubre de 2004, por la que se dictan instrucciones complementarias del contenido de la Orden de 1 de octubre de 2003, que establece criterios para la aplicación de la evaluación y promoción, y requisitos para la titulación del alumnado que curso Educación Secundaria Obligatoria (BOC n ${ }^{\circ} 227$ de 22 de noviembre de 2004).

Circular 1/2005, de 2 de marzo de la D.G. O. Y Evaluación Educativa, por la que se compendia la normativa vigente sobre evaluación de alumnado en E.S.O. (C.A. de Andalucía, matiza las Instrucciones de 21 de noviembre de 2003)

Resolución de 10 de enero de 2005, de la Dirección General de Política Educativa, por la que se prorrogan y modifican parcialmente las instrucciones de 28 de noviembre de 2003 sobre evaluación, promoción y titulación en la ESO. (BOA nº145 de 14 de enero de 2005) (CA de Aragón).

Orden de 7 de marzo de 2005, por la que se establece la regulación de programas de diversificación curricular en la Educación Secundaria Obligatoria $\left(\mathrm{BOC}^{\circ} 055\right.$ del 17 de marzo de 2005).

Orden de 10 de agosto de 2005, por la que se aprueban las instrucciones de organización y funcionamiento de los Institutos de Educación Secundaria dependientes de la Comunidad Autónoma de Canarias. (BOC n ${ }^{\circ} 169$ de 29 de agosto de 2005).

Ley Orgánica 2/2006, de 3 de mayo, de Educación. (BOE nº 106, de 4 de mayo de 2006).

Orden de 28 de julio de 2006, por la que se aprueban las instrucciones de organización y funcionamiento de los Institutos de Educación Secundaria dependientes de la Consejería de Educación, Cultura y Deportes del Gobierno de Canarias (BOC n ${ }^{\circ} 161$ de 18 de agosto de 2006).

Decreto 127/2007, de 24 de mayo, por el que se establece la ordenación y el currículo de la Educación Secundaria Obligatoria en la Comunidad Autónoma de Canarias. (BOC n ${ }^{\circ} 113$ de 7 de junio de 2007).

Orden de 7 de noviembre de 2007 por la que se regula la evaluación y promoción del alumnado que cursa la enseñanza básica y se establecen los requisitos para la obtención del título de graduado en Educación Secundaria Obligatoria (BOC ${ }^{\circ}$ 235 del 23 de noviembre de 2007).

\section{Francia}

Décret n87-32 du 23-1-1987 instituant le diplôme national du brevet, modifié par le décret n²005-1010 du 22-8-2005 (JO du 25-8-2005; BO n³1 du 1-9-2005) qui prévoit la délivrance du diplôme avec des mentions, 
Décret n²006-533 du 10-5-2006 (JO du 12-5-2006; BO n²2 du 1-6-2006) relatif à la note de vie scolaire.

Décret n $96-465$ du 29-5-1996 (JO du 31-5-1996; BO n²5 du 20-6-1996) relatif à l'organisation de la formation au collège (Article 6), modifié par le décret $\mathrm{n}^{\circ} 2005-1013$ du 24-8-2005 (JO du 25-8-2005; BO n³1 du 1-9-2005) relatif aux dispositifs d'aide et de soutien pour la réussite des élèves au collège,

le décret n²006-533 du 10-5-2006 (JO du 12-5-2006; BO n²2 du 1-6-2006) relatif à la note de vie scolaire.

Arrêté du 18-8-1999 (JO du 4-9-1999; BO n³1 du 9-9-1999) relatif au DNB, modifié par l'arrêté du 28-7-2000 (JO du 22-9-2000; BO n³5 du 5-10-2000),

Arrêté du 28-7-2005 (JO du 28-8-2005; BO n³1 du 1-9-2005) relatif aux modalités d'attribution du diplôme national du brevet (il définit les modalités de prise en compte des enseignements de découverte professionnelle),

Arrêté du 1-6-2006 (JO du 14-6-2006; BO n²6 du 29-6-2006) qui prévoit la prise en compte des résultats acquis par les élèves en classe de $3 \mathrm{e}$ uniquement et l'attribution d'une note de vie scolaire.

Arrêté du 10-5-2006 (JO du 12-5-2006; BO n²2 du 1-6-2006) relatif aux conditions d'attribution d'une note de vie scolaire.

Circulaire n²006-105 du 23-6-2006 (BO n²6 du 29-6-2006) relative à la note de vie scolaire.

Arrêté du 30-1-1987 (BO n5 du 5-2-1987) relatif au formulaire d'inscription au DNB.

Note de service n99-123 du 6-9-1999 (BO n³1 du 9-9-1999) relative aux modalités d'attribution du DNB.

Note de service $\mathrm{n}^{\circ} 2000-229$ du 15-12-2000 (BO n ${ }^{\circ} 46$ du 21-12-2000) relative aux modalités d'attribution du DNB à compter de la session 2001.

\section{Inglaterra}

The Education Act 2002, and in the following Regulations:

The Education (England) Regulations 2002 (SI 2002 No.2896)

The Education (School Information) (England) Regulations 2002 (SI 2002 No. 2897)

The Education (England) Regulations 2002 (SI 2002 No 2898)

The Education (England) Regulations 2002 (SI 2002 No. 2899)

The Education (England) Regulations 2002 (SI 2002 No. 2900) 
The Education (England) Regulations 2002 (SI 2002 No. 2901)

The Education (Co-ordination of Admission Arrangements) (Secondary Schools)

(England) Regulations 2002 (SI 2002 No. 2904)

\section{PROFESIOGRAFÍA:}

\section{Ángel José Rodríguez Fernández}

Departamento de Educación ULPGC. Profesor de: Nuevas tecnologías Aplicadas a la Educación; Organización, Procesos e Innovación Educativa; Didáctica General; y Aspectos Organizativos y Didácticos de la Educación Especial

Algunas Publicaciones:

- «Metaevaluación de la ESO en Canarias», Tesis de la ULPGC Dpto. de Psicología y Sociología, marzo 2009.

- Visión Diacrónica de la Supervisión educativa y propuesta de un modelo alternativo (1992). Revista El Guiniguada. ULPGC

- Desarrollo de la expresión y comprensión oral (1995). Revista de la Consejería de Educación del Gobierno de Canarias «Cuadernos de aula»

- «La evaluación en el marco de la educación infantil y primaria. Evaluación del proceso de enseñanza y aprendizaje. Función de la evaluación. Estrategias, técnicas e instrumentos de evaluación» (2000). ICSE Oposiciones a la función docente. Tomo I

- «La evaluación en la educación secundaria. Evaluación del proceso de enseñanza y aprendizaje. Función de los criterios de evaluación. Procedimientos e instrumentos de evaluación. La promoción en la ESO. (2000). ICSE Oposiciones a la función docente. Tomo II.

— «Evaluación de las CCBB en la Enseñanza Obligatoria» (2011). Conferencia Ibérica ieTIC. IP Bragança y Universidad de Salamanca.

- «Estudio preliminar de la influencia de las TIC, televisión y consola en la percepción visual y la sociabilidad». (2011). Conferencia Ibérica ieTIC. IP Bragança y Universidad de Salamanca. 


\section{José Mario Hernández, Pérez.}

Universidad de Las Palmas de Gran Canaria. Departamento de Educación Física ULPGC. Profesor de: Deportes alternativos; y Fundamentos del Deporte (balonmano, hockey y fútbol).

Algunas Publicaciones:

- «Educación física y raciovitalismo: análisis de contenido de los currículos de la ESO», Tesis de la ULPGC Dpto. de Educación Física, junio de 2003.

- «Las tres grandes metáforas de la Educación Física (2002). Revista digital efdeprotes. http://www.efdeportes.com/

— «Apuntes sobre el discurso deportivo de Ortega y Gasset. (2006). Revista Española de Educación Física y Deportes. No 4 .

- En torno a la deportividad en la Educación Secundaria (2006). Revista Tamadaba.n ${ }^{a}$ 9.

- Análisis del juego de ataque en balonmano femenino (2010). Revista Ágora para la Educación Física y el Deporte. No 12 (3).

\section{Dr. José Juan Castro Sánchez.}

Universidad de Las Palmas de Gran Canaria. Profesor de: Métodos de investigación en Educación (Psicopedagogía); Diagnóstico en Educación

Algunas publicaciones:

- Estudios sobre la calidad educativa

— «La metaevaluación en la ESO, análisis y propuestas» (2009), tesis ULPGC.

Datos de contacto: Universidad de Las Palmas de Gran Canaria, Facultad de Formación del Profesorado, Departamento de Educación, Edificio de Formación del Profesorado. C/ SANTA JUANA DE ARCO,1, 35004-Las Palmas de Gran Canaria. Despacho 113, Email: arodriguez@dedu.ulpgc.es

Fecha de recepción: 23 de octubre de 2011

Fecha de revisión: 2 de diciembre de 2011 y 23 de febrero de 2012

Fecha de aceptación: 1 de abril de 2012 